\title{
Occasions manquées pour une réforme globale du secteur de la sécurité au Mali
}

\author{
Zeïni Moulaye \\ Conseiller des Affaires étrangères et ancien ministre des Transports \\ et du Tourisme du Mali
}

\section{Introduction}

Depuis la révolution démocratique de mars 1991, le Mali a été considéré comme un "exemple» voire un «modèle» de gouvernance démocratique en Afrique de l'Ouest, y compris en matière de gestion transformationnelle du secteur de la sécurité. Mais les événements de 2012 ont ébranlé cette certitude. Cette année-là a vu successivement la résurgence de la rébellion touarègue en janvier 2012, un coup d'Etat en mars 2012 contre un pouvoir taxé par les putschistes «d'incompétent» (Konaté 2013: 252), et l'entrée en scène de mouvements djihadistes islamistes, en avril 2012. Lalliance momentanée de la rébellion, des djihadistes et d'autres acteurs de la criminalité transnationale (Moulaye 2014) tels que les trafiquants de drogue a conduit à l'occupation par ces mouvements armés des régions du Nord du pays, soit les deuxtiers du territoire national. Pendant près d'un an, ces criminels de tout acabit ont pillé, volé, violé, détruit et commis avec une violence inouïe des atteintes graves aux droits humains. Ces événements ont mis en lumière le déficit de gouvernance globale au Mali - dans les domaines politique, économique, social, culturel et sécuritaire.

Des défaillances graves sont notamment apparues dans le domaine de la sécurité et celles-ci nont pas pu être comblées par le processus de réforme du secteur de la sécurité timidement amorcé en 2005 par le gouvernement du général Amadou Toumani Touré. Pourquoi? A coup sûr parce la réforme a

Comment citer ce chapitre du livre:

Moulaye, Z. 2015. Occasions manquées pour une réforme globale du secteur de la sécurité au Mali. Dans: Bryden, A et Chappuis, F (dir. publ.) Gouvernance du secteur de la Sécurité : Leçons des expériences ouest-africaines, Pp. 85-102. London: Ubiquity Press. DOI: http://dx.doi.org/10.5334/bav.e. Licence: CC-BY 4.0. 
manqué de leadership, de portage politique et d'appropriation. Il semble que ces déficits essentiels découlent d'un conservatisme d'arrière-garde de certains officiers de la hiérarchie arc-boutés sur des privilèges souvent immérités (en termes de recrutements, de grades, de postes, d'indemnités). Ces passe-droits risquaient, en effet, dêtre remis en cause par une réforme qui privilégierait une bonne gouvernance du secteur de la sécurité, un contrôle démocratique, la transparence dans la gestion, lefficacité et l'obligation de rendre des comptes. Cette situation s'explique avant tout par l'absence de volonté politique au plus haut niveau de l'Etat, le chef de l'Etat étant constitutionnellement le chef suprême des armées et, de surcroît, un ancien officier général.

Il nous semble que l'histoire du Mali moderne a eu des opportunités exceptionnelles pour repenser la sécurité et réformer le secteur de la sécurité. Cela a été le cas, en tout premier lieu, au moment de la proclamation de l'indépendance, le 22 septembre 1960. A cette époque, les nouvelles autorités maliennes ont fait appel à tous les ressortissants du pays servant dans les rangs de l'armée française et au sein des services de sécurité coloniaux pour qu'ils rejoignent leur patrie nouvellement indépendante et forment "une armée malienne» ainsi que des "services de sécurité maliens». Cependant, la sécurité, en tant que concept et pratique, n’a pas été repensée en fonction des nouvelles réalités politiques, économiques, sociales et culturelles du Mali naissant. Les forces de défense et de sécurité (FDS) ont été créées au lendemain de l'indépendance sans que les membres qui les composaient n’aient été «reformatés». Or, durant la période coloniale, ces troupes avaient été «formées» pour servir l'administration française contre les populations colonisées qui venaient de déclarer leur indépendance. De ce fait, sans le vouloir, les forces qui se sont mises à la disposition du nouveau régime étaient dotées de la même mentalité (de domination) et du même comportement (répressif) que ceux existant au sein des troupes coloniales. A leur décharge, il est fort probable que cette situation ait été voulue par les nouveaux gouvernants qui cherchaient ainsi à mieux asseoir leur autorité et leur puissance de domination, ce qui a entraîné plus tard l'avènement d'un régime dictatorial.

Une seconde opportunité de repenser la sécurité et de réformer le secteur de la sécurité s'est présentée au moment de l'avènement de lère démocratique, le 26 mars 1991. En 1990 déjà, la rébellion dans le Nord du Mali avait révélé l'ampleur de la prolifération des armes légères et de petit calibre ainsi que leurs dégâts immenses sur la sécurité humaine. En 1991, lors de la révolution démocratique, les forces de sécurité ont été vilipendées, taxées de «forces de répression à la solde d'un dictateur». Ces forces ont été vouées aux gémonies, au point que, durant les journées agitées du mois de mars (du 22 au 26) et même quelques semaines plus tard, aucun policier ne pouvait porter sa tenue dans la rue sans être livré à la vindicte populaire. Ce changement de régime a offert l'occasion d'opérer un changement qualitatif dans la gouvernance de la sécurité en intégrant celle-ci dans la gouvernance démocratique globale du pays. Au lieu 
de cela, les forces de sécurité, particulièrement la police, ont été violemment prises à partie et marginalisées au motif quelles avaient agi comme des forces de répression au service d'un régime dictatorial. Pire, la défense et la sécurité ont été négligées, reléguées au second plan et dotées d’un budget dérisoire.

Vingt ans plus tard, le Mali a chèrement payé cette négligence. Déjà au début des années 2000, face à la montée de la criminalité transfrontalière et transnationale organisée, les citoyens, les communautés ainsi que les élus locaux et nationaux ont adressé de multiples questions et interpellations aux autorités à propos de la sécurité. Mais, faute d'une réforme suffisamment profonde, dix ans plus tard, en 2012, des hordes de narcoterroristes alliés à des bandes d'irrédentistes ont fait s'effondrer lédifice institutionnel et démocratique en quelques jours. Les forces de défense et de sécurité ont été incapables de contenir ces ennemis de la République, faute de formation adaptée, déquipements et surtout de motivation.

Dans la première partie de cette étude, nous allons évoquer létat de la gouvernance de la sécurité avant le lancement de la réforme de 2005. Dans une deuxième partie, nous décrirons les étapes du processus de changement de politique tel qu'il a été conduit jusqu’aux événements de janvier 2012. Dans la troisième partie, nous aborderons le jeu des acteurs et les enjeux de la réforme. Dans la quatrième partie enfin, nous passerons en revue quelques résultats engrangés et nous suggérerons des propositions visant à créer les meilleures conditions possibles afin de poursuivre et approfondir une réforme qui s'impose aujourd'hui plus que jamais au Mali. En conclusion, nous examinerons la durabilité du changement et la perspective de réforme du secteur de la sécurité au Mali.

\section{Etat de la gouvernance du secteur de la sécurité avant la réforme}

Le système de gouvernance de la sécurité au Mali est un vieil héritage colonial dont il a cumulé toutes les tares pendant un demi-siècle. En effet, pendant longtemps, le secteur de la sécurité a souffert d'une absence de leadership, de vision, de stratégie, de communication et de bonne gouvernance. Jusqu'en 2009, les autorités n’avaient adopté aucun document officiel de politique publique de sécurité et avaient encore moins entamé une réflexion sur déventuels changements fondamentaux de paradigmes de gouvernance de la sécurité. Durant cinquante ans, les questions de sécurité ont été exclues du débat public et du contrôle démocratique de sorte que, pour tous les citoyens, la sécurité était un domaine exclusivement réservé aux services de sécurité. Cela a entraîné un désintérêt total du peuple pour la gouvernance de ce secteur. Cette situation sest avérée, au fil du temps, fort préjudiciable à l'efficacité des services de sécurité, notamment lorsque ceux-ci ont eu besoin de la collaboration de la population 
dans la lutte contre l'insécurité et la criminalité organisée. Par ailleurs, bien qu'ils existent formellement, les mécanismes de contrôle des services de sécurité nont jamais fonctionné efficacement, à cause probablement du caractère exclusif, sensible et souverain du secteur de la sécurité.

Le cadre institutionnel malien fait une nette distinction entre la défense et la sécurité. Depuis 1992, il existe deux départements ministériels distincts: le ministère de la Défense et des Anciens combattants et le ministère de la Sécurité intérieure et de la Protection civile. La Défense a uniquement sous sa responsabilité les forces armées. La Sécurité regroupe la police et la protection civile qui ont un statut civil ainsi que la gendarmerie et la garde nationales qui ont un statut militaire. La gendarmerie et la garde nationales sont statutairement gérées par le ministère de la Défense mais elles sont mises à la disposition du ministère de la Sécurité. Cette combinaison des forces vise en réalité à combler le déficit de ressources humaines, matérielles et financières.

Les institutions de la sécurité fonctionnent conformément au droit international. Les services de sécurité ont été créés conformément à la législation et à la réglementation en vigueur au Mali (lois, décrets, arrêtés ministériels, décisions administratives, etc.). Ils obéissent au pouvoir civil démocratiquement mis en place. Le budget alloué à la sécurité est planifié dans le cadre du budget national et soumis à l'approbation du Conseil des ministres et de l'Assemblée nationale. Les dépenses des services de sécurité ne relèvent d'aucun secret d'Etat. Elles sont accessibles à tous et leur gestion est confiée à des services spécialisés de l'administration publique rattachés au ministère de la Sécurité.

La sécurité au Mali relève du domaine public. Les missions et les prérogatives des services de sécurité sont déterminées par la loi fondamentale. ${ }^{1}$ Leur fonctionnement et leur gestion sont assurés par des mécanismes institutionnels. Dans la pratique, le cadre de gouvernance de la sécurité ne peut pas ignorer certains mécanismes endogènes de gestion de la sécurité dans le Mali profond. Dans le milieu rural en particulier, les communautés font plus souvent appel, dans le processus de gouvernance locale de la sécurité, aux us et coutumes et aux autorités traditionnelles (chefferies, cadis, imams, griots, etc.). C'est là l'un des enjeux nouveaux de la gouvernance démocratique de la sécurité au Mali.

Le contrôle direct des services de sécurité est dévolu à l'Assemblée nationale qui, à travers le contrôle de laction gouvernementale, peut à tout moment interpeller les responsables de la sécurité. Il y a, au sein du Parlement, une «Commission défense, sécurité et protection civile» qui est habilitée à connaître des dossiers de sécurité, à les recevoir, à les analyser et à prendre les décisions qui s'imposent légalement. Elle peut, au besoin, interpeller le ministre et d’autres responsables de la sécurité sur un ou plusieurs points concernant le secteur de la sécurité.

Par ailleurs, plusieurs dispositions constitutionnelles confèrent au président de la République, chef suprême des armées, un contrôle politique sur les forces armées et de sécurité. ${ }^{2}$ Le président de la République préside le Conseil supérieur de la défense nationale et le Comité de défense de la défense nationale 
qui sont respectivement des organes de direction de politique générale en matière de défense, de direction militaire et de gestion de crise.

Le pouvoir judiciaire dispose également de prérogatives constitutionnelles et légales pour contrôler le secteur de la sécurité. Aux termes du Code de procédure pénale, c'est la police judiciaire, composée d’officiers de police judiciaire, qui est chargée notamment de constater les infractions en matière de crimes et de délits, d'en rassembler les preuves et d'en rechercher les auteurs. Les officiers de police judiciaire sont des auxiliaires de justice qui travaillent sous le contrôle et la responsabilité du procureur de la République. Le pouvoir judiciaire dispose aussi de mécanismes de contrôle des services de sécurité par le biais de dispositions de la loi martiale ainsi que des tribunaux et cours militaires.

De fait, l'existence de tous ces mécanismes institutionnels de contrôle n'a pas empêché les dysfonctionnements, les dérapages, les déficits et défaillances constatés en 2012. Pourquoi? Parce qu’en vérité le contrôle parlementaire au Mali se limite le plus souvent à l'adoption du budget de la sécurité, à l'examen de projets de lois et, très rarement, à des questions interpellant le ministre de la Sécurité. Les initiatives des députés notamment en termes de propositions de lois sont inexistantes dans ce domaine. Ce phénomène peut s'expliquer d'abord par l'ignorance qui entoure les questions relatives au secteur de la sécurité mais également par le fait que, pour les parlementaires, comme pour le commun des mortels, la sécurité est un «domaine réservé». Il faut ajouter à cela un déficit chronique de communication gouvernementale en matière de sécurité et un manque patent de volonté politique de soumettre le secteur de la sécurité à un contrôle démocratique.

Depuis le début du $21^{\mathrm{e}}$ siècle, le pays a connu une insécurité grandissante. Outre les risques et les vulnérabilités, les menaces les plus préoccupantes sont celles induites par la criminalité organisée: trafic de drogue, d'armes et d'êtres humains, cybercriminalité, terrorisme. Les impacts de ces activités criminelles sont incommensurables sur la sécurité humaine, la cohésion sociale, le respect des droits humains, la stabilité politique et les efforts de construction démocratique et de développement. Les failles dans la surveillance et la prestation des services de sécurité ont accru la vulnérabilité du pays.

Les défis auxquels est confrontée la gouvernance du secteur de la sécurité sont de deux ordres: il y a, d'une part, les défis sécuritaires physiques, nombreux et multiformes et, d'autre part, les défis abondants et variés de gouvernance du secteur de la sécurité.

Parmi les défis liés à la gouvernance, on note un déficit de leadership, de vision, de stratégie, de communication, d'approche de gestion des ressources; une insuffisance des effectifs, de la formation et de l'équipement; une absence de cadre de coordination des actions des forces de sécurité et de cadre de concertation avec les autres acteurs du secteur de la sécurité; et une inadaptation des stratégies aux nouvelles formes de criminalité, en particulier, la cybercriminalité ainsi que la criminalité transfrontalière et transnationale. Tout cela met en évidence le besoin 
d'une nouvelle forme de gouvernance du secteur de la sécurité qui transformerait la sécurité en un facteur de cohésion sociale, d'unité nationale et de développement. Pour ce faire, certains paradigmes fondamentaux de la sécurité doivent être modifiés mais il faut, pour cela, que les esprits soient prêts à accepter lidée du changement.

Comment, alors, concilier l'exigence d'une participation démocratique avec l'exclusivité des services de sécurité? Comment faire en sorte que les parlementaires, les communautés, les organisations de la société civile et parfois les citoyens, contribuent à la création d'un climat de sécurité au moment précis où les forces de sécurité ne sont plus en mesure de faire pleinement face aux multiples défis sécuritaires internes et externes? La réponse est dans l'intégration de la sécurité dans la gouvernance démocratique globale du pays et la promotion d'une nouvelle forme de gouvernance qui autorise un contrôle direct (parlementaire) et indirect (communautaire et citoyen) des services de sécurité afin d’améliorer la qualité du cadre de gouvernance du secteur de la sécurité.

\section{Processus de changement: vers une réforme du secteur de la sécurité}

\section{L’amorce de l'esprit du changement dans les politiques de sécurité}

C’est à la suite du traumatisme subi lors des événements de 1991 que les services de sécurité (et de police en particulier) ont commencé à réfléchir à la nature de leurs tâches et à leur devenir. Mais ce n'est qu’en 2001 qu’un débat structuré a été lancé dans le cadre des «Journées de réflexion de la police nationale». Ces Journées ont été organisées du 21 au 23 février 2001 à Bamako à l'initiative de la Direction générale de la police nationale. Certains problèmes auxquels étaient confrontées les forces de sécurité ont alors été examinés, tels que les ressources humaines, matérielles, logistiques, financières ainsi que les infrastructures. Les participants à ces Journées ont également abordé les éventuels contours du «nouveau visage» de la police, une police «présente pour renforcer le sentiment de sécurité dans la cité; respectueuse des lois et des droits de la personne dans l'exécution de ses missions; discrète pour mieux s'informer, communiquer avec les citoyens, prévenir les crimes et délits et améliorer l'efficacité de ses prestations dans la cité; tolérante des concepts traditionnels et socioculturels de son milieu pour préserver l'harmonie sociale au sein de la famille et de la communauté; disciplinée dans l'organisation et l'exécution des missions traditionnelles et spécifiques de sécurité publique; forte pour assurer la protection des personnes et des biens, combattre le banditisme et la criminalité, conformément aux dispositions juridiques, administratives et réglementaires en vigueur" (DGPN 2001: 4).

En 2003, le secrétaire général du ministère de la Sécurité, l’inspecteur général de police, Anatole Sangaré, a présenté les grandes orientations de son 
ministère à une mission du Centre deétudes stratégiques du Nigéria, venue à Bamako pour s'enquérir de l'expérience malienne de gouvernance de la sécurité. Ces orientations visaient notamment à privilégier la politique de prévention; asseoir les bases d'une politique de proximité; clarifier et harmoniser les responsabilités en matière de sécurité; adapter les moyens à la réalité; consolider et renforcer l'ouverture sur l'extérieur; et promouvoir une stratégie de communication conséquente. Sur cette base, le ministère envisageait l'adoption d'une politique nationale de sécurité «pragmatique et cohérente dans un environnement caractérisé par des exigences apparemment contradictoires: d'une part la nécessité de la préservation de l'ordre public et de la stabilité de l'Etat; d'autre part la nécessité du respect de l'Etat de droit, des droits fondamentaux de la personne humaine et de notre tradition de tolérance et d'hospitalité» (MSIPC 2003a: 7).

Pour mettre en œuvre ces orientations, le ministère a esquissé un plan opérationnel couvrant sept domaines prioritaires d'intervention: le renforcement de la sécurité des personnes et des biens; le renforcement des capacités opérationnelles des forces de sécurité; une meilleure adaptation du dispositif de couverture sécuritaire; l'adaptation de l'environnement juridique; la mise en place d'une véritable politique de communication; l'amélioration des méthodes de gouvernance; la promotion d'une politique de coopération bilatérale et multilatérale (MSIPC 2003b : 4).

Le processus des «Journées de réflexion de la police nationale» n'a pas abouti à un projet de réforme du secteur de la sécurité. Cela peut s'expliquer soit par un effet d'autocensure des forces de sécurité, soit par une absence d'aval de la hiérarchie, nécessaire pour aller de l'avant. La police est régie par une structure verticale où même la réflexion doit être impulsée par la superstructure. L’opportunité d’évoluer vers une réforme ne s'est à nouveau présentée qu’en 2005, soit quatre années plus tard. Déjà en 2003, les signes d'un éveil de la hiérarchie aux valeurs et critères de bonne gouvernance du secteur de la sécurité sétaient faits sentir, mais ces éléments n'avaient pas encore été partagés avec la base, et encore moins avec les autres agences dans le secteur de la sécurité. En outre, le concept de réforme du secteur de la sécurité n’avait pas encore effleuré les esprits. Néanmoins, les acteurs centraux ont commencé à réaliser la nécessité d’évoluer vers le changement tant souhaité par les populations. Il ne manquait qu'un élément déclencheur pour que le processus se mette en marche. En fait, plusieurs feux rouges sétaient allumés et d'autres signaux allaient apparaître de manière plus éclatante et parfois foudroyante. C’est dans ce contexte que les événements de mars 2005 ont servi de catalyseur pour une nouvelle tentative de réforme.

\section{Les facteurs déclencheurs du processus de réforme}

Le 27 mars 2005, un événement qui aurait pu être banal a entraîné une onde de choc. Un match de football comptant pour les éliminatoires de la Coupe 
d'Afrique des nations a vu la victoire de l'équipe du Togo sur celle du Mali. Dans la nuit, Bamako, la capitale, a été occupée jusqu’à l'aube et mise à sac par une horde de hooligans auxquels se sont joints des milliers de jeunes gens. Le service d'ordre a été plus que défaillant voire totalement absent laissant les populations livrées à leur sort. Cette réaction violente était une revanche de la jeunesse désœuvrée sur les soucis du quotidien ambiant (chômage endémique, pauvreté, misère, marginalisation, exclusion). Elle marquait aussi une défiance envers l'autorité de l'Etat accusé par ces populations d'être à l'origine de leurs malheurs et un dégoût de la gestion gouvernementale. Par sa spontanéité et son degré de violence, lévénement a surpris tout le monde, y compris le président de la République. ${ }^{3}$ Le 7 avril 2005, le premier ministre a rencontré la société civile qui lui a exprimé son exaspération et a réclamé la mise en place d'un Etat qui garantisse la sécurité des populations et de leurs biens, un Etat capable de fournir l'assistance requise au moment nécessaire, un Etat fort qui n'implique cependant pas un retour à la dictature. Cette situation a clairement mis en lumière au moins trois défaillances systémiques dans la gestion du secteur de la sécurité:

- un dysfonctionnement lié à la déliquescence de l'autorité de l'Etat et un déficit de communication, ce qui nécessitait un diagnostic approfondi du secteur de la sécurité;

- un système de gestion gouvernementale où très souvent les lauriers étaient mieux partagés que les responsabilités;

- un manque conséquent de responsabilité et d’efficacité des forces de sécurité publique et des moyens qui leur étaient alloués.

Au lendemain de cet événement, des responsables de la sécurité ont été relevés de leurs fonctions sans que cela n'apaise l'opinion publique, qui a unanimement réclamé un changement de la situation sécuritaire. Cependant, les acteurs du secteur de la sécurité n'avaient pas tous le même degré de conscience ni la même vision du changement. Au sein des services de sécurité, beaucoup croyaient qu'il suffirait de réaménager les textes existants et d'améliorer les conditions de travail et de vie des agents de sécurité pour régler ce malaise. Pour une poignée d'entre eux cependant, ainsi que pour une bonne partie des acteurs de la société civile, la situation sécuritaire appelait une nouvelle gouvernance de la sécurité afin de répondre aux exigences démocratiques et affronter les menaces sécuritaires majeures qui se manifestaient sur le terrain : trafics de drogue, d'armes et d'êtres humains, catastrophes naturelles, présence de salafistes algériens sur le territoire national, etc.

C’est dans ce contexte que le ministère de la Sécurité intérieure et de la Protection civile a initié, en 2005, les «Etats généraux de la sécurité et de la paix au Mali ». 


\section{Les étapes importantes de mise en œuvre et l'évolution du processus de la réforme}

Lidée des «Etats généraux de la sécurité et de la paix au Mali»a émané du ministère de la Sécurité. Cette rencontre a constitué le point d'entrée dans le processus de réforme du secteur de la sécurité et a été un moment historique dans les annales de la sécurité au Mali. Pour la première fois depuis l'indépendance, en 1960, les services de sécurité s'ouvraient aux autres acteurs du secteur de la sécurité. Tous les problèmes ont pu être évoqués et débattus sans tabou. Il $\mathrm{y}$ avait là des officiers généraux et des paysans, des soldats et des chasseurs, des policiers et des éleveurs, de hauts fonctionnaires et des ouvriers, des femmes, des hommes, des jeunes gens et des personnes âgées. Tous les participants ont identifié ensemble les défis sécuritaires, évalué les besoins de sécurité des populations et proposé des solutions ainsi que la forme de gouvernance nationale de la sécurité qu’ils appelaient de leurs vœux.

Les «Etats généraux de la sécurité et de la paix au Mali » se sont déroulés du 21 au 23 novembre 2005, sous la présidence effective du président de la République, Amadou Toumani Touré, et sous l'égide du ministère de la Sécurité intérieure et de la Protection civile. La préparation de cet événement a été séquencée en trois phases. Dans une première phase, le ministère a envoyé des missions de sensibilisation sur le terrain, parfois jusqu'aux communes et aux villages, pour faire prendre conscience aux divers acteurs de la nécessité d'un débat national sur les questions de sécurité et pour recueillir leurs réactions. Durant la deuxième phase, le ministère a organisé des concertations régionales sous la responsabilité des gouverneurs de région autour de thématiques centrales comme le banditisme urbain et périurbain, la prolifération des armes légères, la gestion des conflits communautaires, la montée de l'intolérance religieuse, la criminalité transfrontalière, etc. La troisième phase a été la tenue, à Bamako, des Etats généraux qui ont regroupé 245 participants venus d'horizons divers et représentant le gouvernement, l'administration générale, les régions administratives, les commandements de régions militaires, la défense et la sécurité, la société civile, le secteur privé, les partis politiques, les collectivités locales, les communautés ainsi que des partenaires techniques et financiers, etc.

Quatre objectifs ont été fixés à ces Etats généraux de la sécurité et de la paix:

- inciter toutes les composantes de la nation à participer activement à un débat sur les questions de sécurité et à la conception d’un programme cohérent et harmonisé de sécurité;

- identifier les éléments d'une «Politique nationale de sécurité» à travers l'examen, sous divers angles, des problèmes de sécurité;

- réunir les éléments d'un projet de loi d’orientation et de programmation en matière de sécurité; 
- mettre en place un cadre de concertation, de coordination et de gestion participative des questions de sécurité entre tous les acteurs du secteur de la sécurité.

Afin d'atteindre ces objectifs, trois jours durant, les participants ont alterné des séances plénières et des ateliers assortis de témoignages de hautes personnalités, mais aussi de citoyens ordinaires qui ont raconté leur vécu de la sécurité au Mali. La conférence inaugurale a été axée sur la problématique sécuritaire en Afrique de l'Ouest; elle a été suivie de 21 autres communications qui ont porté sur des sujets d'actualité. Les travaux en ateliers ont été articulés autour de cinq grands thèmes: les sociétés du 21 e siècle et l'émergence de nouveaux risques; le principe de précaution et la culture de la prévention; la sécurité et la décentralisation à l'horizon 2015 ; la sécurité et les conflits intercommunautaires; les acteurs de la sécurité face à leurs responsabilités. Chaque atelier a été animé par un modérateur et les débats ont été recensés par un rapporteur qui travaillait en relation étroite avec le rapporteur général.

Sagissant particulièrement de la vision de la sécurité, deux thèses ont été émises lors de ces Etats généraux. La plupart des forces de sécurité ont appelé au renforcement de l'autorité de l'Etat et à la restauration d'un Etat fort pour assurer la sécurité. Les autres acteurs, notamment ceux issus de la société civile, de la société politique et des collectivités locales (communautés), largement majoritaires, ont prôné une vision nouvelle qui reposerait sur une gouvernance de la sécurité plus démocratique où lêtre humain serait au centre des préoccupations sécuritaires. Nombre de communications et d'interventions ont plaidé afin que cette nouvelle vision soit fondée sur la sécurité humaine (MSIPC 2005). Les partisans de cette thèse dominante avaient la conviction que, si la sécurité était centrée sur une gouvernance démocratique et sur lêtre humain, elle impulserait une nouvelle dynamique au développement. Cette vision partait du constat que, depuis la fin de la guerre froide, les conflits interétatiques ont cessé. Le centre de gravité des menaces extérieures a brutalement basculé de l'extérieur vers l'intérieur révélant ainsi une multitude de vulnérabilités, de risques et de menaces réelles à la paix et à la sécurité au niveau national. Constatant que la violence avait gagné du terrain dans toutes les couches de la société, les tenants de cette thèse ont souligné la nécessité de revoir le schéma classique de la sécurité jusque-là centré sur la protection de l'Etat. En somme, ces individus affirmaient que, désormais, il fallait privilégier la sécurité humaine sans porter atteinte cependant à la sécurité de l'Etat.

Ainsi donc, le 23 novembre 2005, à l'issue des trois jours de débats intenses, francs et directs, 127 recommandations ont été formulées dont les plus importantes sont les suivantes:

- l'élaboration d'une politique nationale de sécurité fondée sur une nouvelle vision (la sécurité humaine) et une nouvelle stratégie globale (la prévention); 
- la mise en œuvre d'un programme de gouvernance partagée de la sécurité et de la paix;

- la conception et la mise en œuvre d'une stratégie de communication pour une gouvernance partagée de la sécurité et de la paix;

- lélaboration (dans la perspective d'une loi de programmation sécuritaire) d'un plan d'action à long terme de mise en œuvre des axes stratégiques de la politique nationale de sécurité.

Ces recommandations ont dessiné une feuille de route destinée à améliorer la qualité démocratique de la gouvernance du secteur de la sécurité au Mali, en identifiant comme première étape la rédaction d'une nouvelle politique nationale de sécurité.

\section{La politique nationale de sécurité et de protection civile}

Au lendemain de la tenue des Etats généraux de la sécurité et de la paix, le ministère de la Sécurité a réuni une commission chargée d'esquisser une politique nationale de sécurité intérieure et de procéder à la mise en place d'un programme de gouvernance partagée de la sécurité dans le cadre du démarrage concret de la réforme du secteur de la sécurité. Ce programme visait à contribuer qualitativement à l'instauration d'un climat de paix sociale et de stabilité politique propice au développement économique et social en intégrant la sécurité dans la gouvernance démocratique et développementale globale du pays. Lobjectif était de répondre aux exigences démocratiques et aux critères de bonne gouvernance et d'améliorer les capacités des forces de sécurité pour faire face aux facteurs internes et externes d'insécurité et répondre aux attentes des populations en matière de sécurité.

Ce document de politique nationale de sécurité intérieure a pris la forme d'un simple énoncé de politique et soulignait le caractère polysémique, transversal et multidimensionnel de la sécurité en déclinant neuf axes stratégiques autour desquels devait être articulée la politique nationale de sécurité et de protection civile:

1. la prévention des situations à risques pour la sécurité;

2. le renforcement des capacités des forces de sécurité;

3. le renforcement des capacités nationales en matière de prévention et de gestion des catastrophes;

4. la lutte contre l'insécurité routière et fluviale;

5. la mise en place de la police de proximité;

6. la mise en œuvre d'une gouvernance partagée de la sécurité;

7. la lutte contre le terrorisme;

8. le renforcement de la coopération bilatérale et multilatérale; 
9. la mise en œuvre d'une stratégie de communication favorisant le changement d'attitudes et de comportements.

A travers ces axes, le gouvernement entendait mener des actions visant à renforcer les capacités de l'Etat à faire face aux problèmes de sécurité dans leur diversité et leur complexité et à assurer à l'ensemble du pays un climat de paix sociale et de stabilité politique propice au développement.

Cet énoncé de politique nationale de sécurité était assorti d'un plan d'action quinquennal qui devait servir de loi de programmation sécuritaire. Ladoption de ce plan d'action a été différée par le Conseil des ministres pour des raisons à la fois budgétaires et politiques. Celui-ci avait estimé que le budget requis nétait pas disponible. En outre, pour le chef de l'Etat en particulier, une loi de programmation sécuritaire devait aller de pair avec une loi de programmation militaire qui nétait pas encore prête. En revanche, l'idée d'un programme qui soutiendrait la mise en œuvre de la politique nationale de sécurité a été retenue: ainsi est né le Programme de gouvernance partagée de la sécurité et de la paix (PGPSP).

\section{Le Programme de gouvernance partagée de la sécurité et de la paix}

La mise en œuvre du PGPSP, comme instrument de mise en œuvre de la politique nationale de sécurité, a donné un coup d’envoi significatif au processus de réforme du secteur de la sécurité (Moulaye et Niakaté 2011). Lobjectif global du programme était de contribuer à l'instauration d'un climat de sécurité, de paix et de stabilité, qui favorise le développement humain durable et soutienne la lutte contre la pauvreté au Mali. Il devait atteindre trois objectifs spécifiques, à savoir: (i) appuyer l'élaboration et l'adoption de la nouvelle politique nationale de sécurité issue des Etats généraux de la sécurité et de la paix; (ii) appuyer la mise en œuvre de la gouvernance partagée de la sécurité et de la paix aux niveaux national et décentralisé; et (iii) appuyer la construction d'une sécurité et d'une paix durables dans le Nord du Mali.

Le programme était articulé autour de quelques activités majeures:

- lélaboration et la mise en œuvre d'un document cadre de politique nationale de sécurité et de protection civile telle que recommandée par les Etats généraux de la sécurité et de la paix au Mali;

- le renforcement des capacités des services de sécurité et de protection civile, des organisations de la société civile, du secteur privé, des collectivités territoriales et des communautés locales en matière de gouvernance de la sécurité;

- la prévention et la résolution des conflits communautaires, en collaboration étroite avec les organisations de la société civile, les collectivités territoriales et les représentants de l'Etat; 
- le renforcement des capacités de prévention et de gestion des catastrophes;

- la conception et la mise en place d'un système pilote de police municipale;

- l'élaboration et la mise en œuvre d'une communication pour une sécurité partagée et décentralisée;

- la promotion de la culture de la paix;

- la maîtrise des armes légères, etc.

Plus largement, ce programme a appuyé plusieurs institutions de la République (Assemblée nationale, Conseil économique et social, ministères de l'Administration territoriale, de la Défense, de la Promotion de la femme, de la Justice, etc.). Il a accompagné techniquement et financièrement de nombreuses communautés, collectivités locales et organisations de la société civile dans leurs actions de prévention, de gestion et de résolution de conflits. Les espaces de dialogue et de concertations créés à cette occasion ont permis d’engager des débats féconds sur les problématiques sécuritaires du pays. Ces échanges ont souvent éclairé les artisans de la paix et les acteurs de la sécurité et leur ont permis d'identifier des résolutions pertinentes qui ont pu contribuer à la consolidation de la paix et de la sécurité dans le Mali profond. Enfin, le programme a tissé un réseau de partenariat qui a contribué utilement à son financement. Ces résultats nous paraissent d'autant plus importants qu'ils touchent à lêtre humain, la plus grande richesse d'une nation. Ils ont permis à certains acteurs de prendre conscience de la nécessité de s'investir dans le domaine de la sécurité, un secteur qui leur était totalement étranger. La conviction et la persévérance aidant, ce programme a permis l'implication d'autres acteurs qui ont constitué une masse critique permettant de rendre cette réforme irréversible.

A l'issue des trois années de mise en œuvre (octobre 2008 - décembre 2011), le programme a engrangé des résultats non négligeables, y compris lélaboration d'un Document cadre de politique nationale de sécurité intérieure et de protection civile. En outre, la réalisation d'un certain nombre détudes a renforcé les bases de la réforme, comme par exemple, une étude de faisabilité sur la police communale; une étude de création d'une base de données sur la criminalité; et une étude relative à la stratégie de communication assortie d'un plan de communication du ministère de la Sécurité intérieure et de la Protection civile.

Il faut inclure dans le bilan positif du PGPSP une centaine d’activités de renforcement des capacités des acteurs du secteur de la sécurité à travers notamment l'organisation de conférences-débats sur les défis sécuritaires au Mali, en Afrique et dans le reste du monde ainsi que sur les réponses institutionnelles et la problématique de la réforme du secteur de la sécurité. Le PGPSP a aussi privilégié des activités de formations, y compris la formation de 170 agents de sécurité en comptabilité matière et en comptabilité publique afin d'améliorer la gestion financière de la sécurité et la formation de 50 agents en sûreté aéroportuaire. L'engagement communautaire a également été un aspect du programme 
qui a inclus l'organisation de 15 rencontres intercommunautaires ou intercommunales et la mise en place d'un projet d'appui au renforcement des capacités de gouvernance locale dans la région de Kidal où se posaient des problèmes spécifiques de sécurité et de développement à la base.

Au-delà de ces résultats, ce programme a initié une dynamique d'implication de la société civile dans la gouvernance de la sécurité, qui sest poursuivie indépendamment des événements qui ont secoué le pays. On note, au niveau des communautés en particulier, une prise de conscience de la nécessité de focaliser les efforts sur la paix et la sécurité comme pré-requis du développement local. En outre les ressources allouées à ce programme ont contribué à atténuer la traditionnelle méfiance entre civils et militaires, à apaiser certaines tensions sociales et à réduire le niveau de violence dans le pays. Cependant, elles se sont révélées insuffisantes au regard des immenses besoins de sécurité et inopérantes face aux nouvelles menaces telles que la criminalité organisée.

Toutes les forces vives de la nation ont été représentées aux Etats généraux en novembre 2005. Elles ont participé au démarrage de la réforme du secteur de la sécurité, mais sans faire forcément preuve de la même détermination. Les organisations de la société civile et les communautés de base étaient les plus enthousiastes. Ces acteurs non étatiques, dont certains voyaient pour la première fois leur statut et utilité reconnus, avaient la conviction que cette réforme pouvait répondre à certaines de leurs préoccupations et se sont investis pleinement dans le débat.

Un an après la tenue de ces Etats généraux, il est possible de constater que la réaction la plus positive à cette initiative est venue de la société civile. En effet, plusieurs organisations de la société civile œuvrant dans les domaines de la paix, de la sécurité et des droits humains se sont regroupées dans une "Coalition nationale de la société civile pour la paix et la lutte contre la prolifération des armes légères (CONASCIPAL) » et ont spontanément décidé d'apporter leur soutien aux efforts du gouvernement pour donner une impulsion à la gouvernance démocratique du secteur de la sécurité. En janvier 2007, la CONASCIPAL a organisé le premier forum national de la société civile sur la gouvernance démocratique de la sécurité au Mali dont les actes ont été publiés (Moulaye 2008). Depuis lors, un peu partout sur le territoire national, les populations ont commencé à manifester un véritable élan d’adhésion aux idéaux de la réforme à travers de multiples actions d'information, de sensibilisation et de formation ainsi que des initiatives de rencontres intercommunautaires et intercommunales.

Lors de ces Etats généraux de la sécurité et de la paix, les forces de sécurité et de défense se sont montrées plus circonspectes mais ne sont cependant pas restées muettes. A leur décharge, elles invoquaient souvent le devoir de réserve et le manque d'habitude d'intervenir en public. En vérité, un grand nombre de membres de ces forces doutaient de la réussite du processus de réforme, ce qui peut paraitre normal car l'apprentissage de la démocratie passe aussi par 
la culture du doute, mais un doute qui doit faire réfléchir et donner ensuite le courage de l'engagement. Il faut tout de même souligner qu'une poignée d'officiers avait, dès le départ, mesuré le poids de l’enjeu et compris la nécessité et les retombées positives de la réforme sur l'avenir du pays. Ils se sont investis dans le processus mais ils étaient trop peu nombreux et noccupaient pas de postes décisionnels. La hiérarchie, elle, a constamment apporté son soutien verbal à cette initiative mais, à l'analyse et a posteriori, il apparaît que ce soutien nétait que de façade. Parmi les acteurs dynamiques figuraient quelques individualités, généralement des chercheurs, dont l'apport a été parfois déterminant en termes d’analyses de la situation sécuritaire, de compréhension des concepts et de lecture des approches.

Pour organiser ces Etats généraux, le ministère a bénéficié de l'appui technique et financier du Programme des Nations Unies pour le développement (PNUD); ce soutien découlait de certaines déclarations du gouvernement malien qui avait fait miroiter la perspective d'ancrer la «sécurité humaine» au cœur de la refondation du secteur de la sécurité. Le PNUD, qui privilégiait à l'époque la dynamique de promotion de ce concept, était au départ l'unique partenaire de ce processus de réforme, mais il a été par la suite rejoint par la Norvège, le Luxembourg et la Suisse qui ont fortement soutenu cette initiative. Au départ, le processus de réforme a bénéficié d'un appui prudent du PNUD, qui a porté notamment sur l'organisation des concertations à l'intérieur du pays et la tenue des Etats généraux de la sécurité. Par la suite, le PNUD a consenti une rallonge budgétaire pour la conception et la mise en place du PGPSP. ${ }^{4}$

\section{Conclusion: La durabilité du changement et les perspectives de consolidation de la réforme}

Quelques semaines après la tenue des Etats généraux, le ministère de la Sécurité a élaboré un Plan d'action quinquennal (2008-2012) destiné à mettre en œuvre tous les aspects "hard» et "soft » de la réforme du secteur de la sécurité dont le budget sélevait à 88,4 milliards de FCFA. Ce plan d'action devait être soumis à l'approbation du gouvernement sous forme de loi de programmation sécuritaire. Les sources de financement prévues étaient le budget de l'Etat et des contributions extérieures au travers d'une table ronde sectorielle. Mais ce projet n'a pas été retenu par le gouvernement au motif qu'il devait être assorti, au préalable, d'une loi de programmation militaire. Cela souligne clairement le manque de volonté politique au plus haut niveau de l'Etat et met en lumière le jeu d'intérêts corporatistes entre la défense et la sécurité. A vrai dire, le gouvernement navait prévu aucune ressource substantielle pour la réforme du secteur de la sécurité, mais s'appuyait plutôt sur le soutien de la table ronde sectorielle, à laquelle ont notamment participé le PNUD et les donateurs externes, ce qui dénote du niveau de volonté politique. 
Ce manque de volonté politique a, en effet, caractérisé le processus de réforme à plusieurs reprises. Ainsi, le Document cadre de politique nationale de sécurité intérieure et de protection civile a connu une longue maturation entre le moment où il a été entamé (janvier 2006) et le moment où il a été adopté par le Conseil des ministres (octobre 2010). Un tel laps de temps dénote soit d'un manque de volonté ferme, soit de tiraillements internes eu égard à son contenu. De la même manière, le Programme de gouvernance partagée dela sécurité et dela paix au Mali a été conçu en 2006, mais sa mise en place n’est intervenue qu’en 2008. Là également, ces deux années de battement laissent à penser que la volonté politique nétait pas suffisamment ferme.

Le président de la République étant lui-même à la fois chef suprême des armées et ancien officier général approuvait-il réellement la réforme du secteur de la sécurité? Pourquoi voulait-il lier la loi de programmation militaire à la loi de programmation sécuritaire? Avait-il peur du décalage entre l'armée et la sécurité qu'une telle réforme aurait entraîné? Sentait-il la réticence de certains officiers au sein de l'armée à toute forme de réforme du secteur de la défense? Savait-il qu'en freinant la loi de programmation, il portait atteinte à tout le processus de réforme du secteur de la sécurité? Voilà des questions que l'on peut légitimement se poser a posteriori.

De ce fait, les efforts de réforme se sont essentiellement limités à la réalisation des activités du PGPSP. Le peu d'enthousiasme du gouvernement pour la réforme a poussé le PNUD à se désintéresser du programme dès que son apport financier a été épuisé. Pendant ce temps, certains acteurs, tels que les organisations de la société civile, les collectivités territoriales et les communautés, ont continué à réclamer l'appui technique et financier de cette agence onusienne, ce qui montrait leur intérêt de poursuivre le processus de réforme.

A l'heure du bilan, il apparaît qu'en interne un travail de fond, en termes de communication, était nécessaire au départ parce que beaucoup d'acteurs au sein des forces de sécurité et de défense - et encore plus au niveau des autres acteurs (société civile, société politique, secteur privé, collectivités locales, communautés) - n'avaient aucune idée de ce que recouvrait une réforme du secteur. Une bonne partie de la hiérarchie sécuritaire n’en voyait pas l'utilité ou en redoutait les conséquences sur leurs privilèges personnels. A l'externe, il y avait l'exigence de mobilisation de fonds pour mener à bien les activités de réforme, mais la plupart des partenaires extérieurs considéraient la sécurité comme un domaine de souveraineté intérieure dans lequel ils ne pouvaient intervenir quà la demande expresse du gouvernement malien. Par ailleurs, les services de sécurité eux-mêmes nont presque jamais bénéficié de programmes de développement financés directement par la coopération internationale. Les forces de sécurité, très peu habituées aux modalités de mobilisation de ressources extérieures, penchaient pour un repli à cause du devoir de réserve. La solution trouvée a été la promotion d'une gouvernance démocratique du secteur de la sécurité afin que la sécurité soit considérée par les partenaires comme 
un domaine ouvert à l'aide publique au développement au même titre que la santé ou l'éducation.

Evidemment, les choses auraient pu prendre un autre tour s'il y avait eu davantage de volonté politique au sommet de l'Etat et un engagement plus fort au niveau de toutes les institutions de la République, si la hiérarchie de la sécurité n'avait pas fait preuve de frilosité, et si dès le départ les forces de sécurité et de défense avaient compris que la réforme du secteur de la sécurité avait un caractère global et sétaient solidarisées pour la mener à bien. En effet, l'idéal aurait été pour le Mali de disposer d'un seul grand programme de réforme fondé sur une vision unique, une stratégie globale, une synergie d'actions et une communication pertinente. Au lieu de cela, plusieurs programmes œuvrant dans les domaines de la paix et de la sécurité ont coexisté sans aucun lien organique ni même une collaboration sur le terrain. Leur fusion aurait remis en cause des intérêts et des ambitions personnels.

Finalement, seule létape de la sensibilisation aux défis sécuritaires, à la nécessité d'une réforme du secteur de la sécurité et à l'exigence de participation de tous les acteurs a été menée de manière relativement satisfaisante. Et seuls les acteurs de la société civile, les collectivités locales et les communautés de base ont montré un véritable intérêt pour la réforme. Il reste donc des pans entiers de la réforme à concevoir et à appliquer, en particulier dans ses dimensions législatives et réglementaires, qui devraient induire une qualité transformationnelle. Le concept de gouvernance partagée de la sécurité s'est frayé un petit chemin. Il reste à l'élargir pour permettre un développement durable soutenant une sécurité fiable ou, mieux encore, ouvrir grande la voie à une sécurité fiable soutenant un développement durable.

A la lumière des tristes événements survenus en 2012, les mentalités ont énormément évolué au Mali. Les forces de sécurité et de défense ont subi une telle humiliation et le peuple malien un tel choc psychologique quaujourd'hui aucune force ne s'oppose à une réforme du secteur de la sécurité. Il reste à en tracer la voie adéquate, la plus intelligente, la plus fiable, la plus efficace et la plus efficiente possible. Le 30 décembre 2013, le ministère de la Sécurité a relancé le processus de réforme par la mise en place d'un «Groupe pluridisciplinaire de réflexion sur la réforme du secteur de la sécurité». De son côté, le ministère de la Défense qui paraissait réfractaire a mis en place une commission de refondation de la défense.

Lidéal serait que ces deux ministères se rapprochent pour mener de concert un processus transformationnel du secteur de la sécurité et faire naître ainsi une vision commune, une politique nationale de sécurité intérieure et une stratégie de défense nationale, qui instaureront un climat apaisé, propice à l'investissement productif et au développement durable. Lancrage d'un tel projet devrait se situer au niveau du président de la République afin de faciliter les arbitrages, la coordination et la synergie d'actions. Cela comblerait d'un coup le déficit de leadership et de portage politique constaté et impulserait la 
dynamique d'appropriation par les acteurs institutionnels qui a fait défaut au cours de la première phase du processus de réforme.

\section{Notes}

${ }^{1}$ La Constitution de la République du Mali a été adoptée le 25 février 1992.

${ }^{2}$ Voir, par exemple, article 44, titre III de la Constitution du 25 février 1992.

${ }^{3}$ Rencontre du président de la République avec la presse (8 juin 2005) à l’occasion du $3^{\mathrm{e}}$ anniversaire de son accession au pouvoir.

${ }^{4}$ Pour le bilan de ce programme se référer à Moulaye et Niakaté (2011). 